\title{
Earth-Ionosphere Cavity Resonances and the Propagation of ELF Radio Waves ${ }^{1}$
}

\author{
James R. Wait \\ National Bureau of Standards, Boulder, Colo. \\ (Received February 3, 1965)
}

\begin{abstract}
This is a tutorial exposition of the theory of ELF propagation in the earth-ionosphere waveguide. Derivations are not given; instead, the plausibility of the results and the physical aspects of certain special cases are discussed. A simplified treatment of the Schumann cavity resonance phenomenon is also given.
\end{abstract}

\section{Introduction}

This is intended to be an introduction to electromagnetic wave propagation in the frequency range below $3000 \mathrm{c} / \mathrm{s}$ and above about $1 \mathrm{c} / \mathrm{s}$. This range has become known as extremely-lowfrequency (ELF) to distinguish it from the very-low-frequency (VLF) band which is now regarded universally as the range $3 \mathrm{kc} / \mathrm{s}$ to $30 \mathrm{kc} / \mathrm{s}$. Attention is confined to propagation in the earth-ionosphere waveguide as this is by far the most important mechanism for the transfer of energy at very great distances from the source. Included in the discussion is a simplified treatment of the earthionosphere cavity resonance phenomenon with special reference to the pioneering work of $\mathrm{W}$. $\mathrm{O}$. Schumann. The influence of nonuniformities and surface irregularities on the cavity resonances is also considered in an appendix.

References to past work in this field will be made at the appropriate place in the paper. However, at this point, the existence of two earlier review articles is mentioned. One is a chapter in a book [Wait, 1962], while the other is a review paper given at a NATO conference over a year ago [Galejs, 1963]. In both these articles, extensive references to related work are given. In addition, there has been a very extensive bibliography, prepared under the auspices of NATO, by Brock-Nannestad [1962]. Therefore, in the present review, only material is referenced which is actually used in the presentation.

\section{Formulation of the Waveguide Problem}

In this section, the salient features of the conventional theoretical approach are given. The model is a homogeneous conducting spherical earth of radius $a$ surrounded by a concentric ionospheric shell of inner radius $a+h$. Thus, $h$ is the height of the ionosphere above the earth's surface. In what follows, it is convenient to introduce the usual spherical coordinate system. Also, unless stated to the contrary, the fields are assumed to vary as exp $(i \omega t)$.

Using the above model, and assuming a vertical electric dipole source located at $\theta=0, r=a$, the expression for the vertical electric field, at $r=a$, is

$$
E_{r}=\frac{I d s \eta}{i 2 k h a^{2}} \sum_{n=0}^{\infty} \delta_{n} \frac{\nu(\nu+1)}{\sin \nu \pi} P_{\nu}(-\cos \theta) .
$$

\footnotetext{
${ }^{1}$ Paper was presented at the ULF Symposium, Boulder, Colo., August 17-20, 1964.
} 
Here, $k=\omega / c$ is the free-space wave number; $\delta_{n}$ is an excitation factor discussed below; $I$ is the average current in the source dipole; $d s$ is the length of the source dipole; $\eta$, the intrinsic impedance of the air space, $\cong 120 \pi ; P_{\nu}(-\cos \theta)$ is the Legendre function of argument $-\cos \theta$ and (complex) order $\nu$.

The complex quantity $\nu$ contains the crux of the propagation problem. It is related to the propagation factor $S_{n}$ for $n=0,1,2, \ldots$ by the relation

$$
\nu+1 / 2=k a S_{n}
$$

The factor $S_{n}$ is determined from the boundary conditions of the earth ionosphere waveguide. At ELF, numerical values of $S_{n}$ may be determined on the basis of a flat-earth model. Assuming for the moment that the earth and the ionosphere are homogeneous isotropic media, it follows from previous work [Wait, 1962] that

$$
S_{n} \cong\left\{1-\left(\frac{\pi n}{k h}\right)^{2} \frac{1}{4}\left[1+\left(1+4 i \frac{\Delta k h}{(\pi n)^{2}}\right)^{1 / 2}\right]^{2}\right\}^{1 / 2},
$$

where

$$
\Delta=\frac{1}{N_{i}}+\frac{1}{N_{g}}
$$

in terms of the refractive indices, $N_{g}$ and $N_{i}$, of the earth and the ionosphere, respectively. This equation is valid subject to the condition $|\Delta| k h<<1$. The sign of the radical is chosen such that the real part of $S_{n}$ is positive and the imaginary part of $S_{n}$ is negative.

For the important case $n=0$, (3) simplifies to

$$
S_{0}=\left[1-i \frac{\Delta}{k h}\right]^{1 / 2}
$$

Furthermore, since $|\Delta| k h /(\pi n)^{2}<<1$, when $n>0$, it follows that

$$
S_{n}=\left[1-\left(\frac{\pi n}{k h}\right)^{2}-i \frac{2 \Delta}{k h}\right]^{1 / 2},
$$

for $n=1,2,3, \ldots$.

When the ionosphere is a homogeneous isotropic medium of conductivity $\sigma_{i}$ and the earth is a homogeneous isotropic medium of conductivity $\sigma_{g}$, it is well known that

$$
N_{i}^{2} \cong \sigma_{i} /\left(i \epsilon_{0} \omega\right) \text { and } N_{g}^{2} \cong \sigma_{g} /\left(i \epsilon_{0} \omega\right)
$$

Thus,

$$
\Delta \cong(1+i)\left(\frac{\epsilon_{0} \omega}{2}\right)^{1 / 2}\left(\frac{1}{\sigma_{i}^{1 / 2}}+\frac{1}{\sigma_{g}^{1 / 2}}\right)
$$

or, in most instances at ELF,

$$
\Delta \cong(1+i)\left(\frac{\epsilon_{0} \omega}{2 \sigma_{i}}\right)^{1 / 2}
$$

because $\sigma_{g}>>\sigma_{i}$. 
It might be mentioned in passing that this simple expression for $\Delta$ is only valid for the idealized model discussed here. However, it is interesting to note that a horizontally stratified ionosphere will give rise to a representation of the form

$$
\Delta=(1+i)\left(\frac{\epsilon_{0} \omega}{2 \sigma_{i}}\right)^{1 / 2} Q
$$

where the complex function $Q$ has been tabulated and graphed for a wide variety of models [Wait, 1962].

\section{Peripheral Waves}

The physical significance of (1) may now be discussed. The Legendre function is well approximated by

$$
P_{\nu}(-\cos \theta) \cong\left(\frac{2}{\pi \nu \sin \theta}\right)^{1 / 2} \cos \left[\left(\nu+\frac{1}{2}\right)(\pi-\theta)-(\pi / 4)\right]
$$

if $|\nu|>>1$ and $\theta$ is not near 0 or $\pi$. Thus, the individual terms (i.e., modes) in the series in (1) are simply proportional to

$$
\frac{1}{(\sin \theta)^{1 / 2}} \cos \left[k a S_{n}(\pi-\theta)-(\pi / 4)\right]
$$

which, apart from a constant factor may be identified as the linear combination of two peripheral waves of the form

$$
\frac{1}{(\sin \theta)^{1 / 2}} \exp \left[-i k a S_{n} \theta\right]
$$

and

$$
\frac{1}{(\sin \theta)^{1 / 2}} \exp \left[-i k a S_{n}(2 \pi-\theta)\right] \exp (i \pi / 2)
$$

It is apparent that these are waves traveling in opposing directions along the two respective great circle paths of respective length $a \theta$ and $a(2 \pi-\theta)$. An interesting feature is the $\pi / 2$ phase advance which the wave traveling on the long path accumulates as it passes through the antipodal point $\theta=\pi$. This, of course, is a characteristic feature of symmetrical waves passing through a perfect focus. It is seen that the phase velocity of these waves or modes of order $n$ is $c / \operatorname{Re} S_{n}$ where $c$ is the velocity of light. Because $\operatorname{Re} S_{n}>1$, the waves are "slow" relative to unimpeded plane waves in free space. The attenuation is given by $-k \operatorname{Im} S_{n}$ in nepers per unit distance apart from geometrical effects which are contained implicitly in the multiplicative factor $1 /(\sin \theta)^{1 / 2}$.

The linear combination of the two peripheral waves forms a standing wave pattern whose distance $\hat{\delta}_{m}$, between minima, is approximately given by

$$
k \hat{\delta}_{m} \operatorname{Re} S_{n}=\pi \text { or } \hat{\delta}_{m} \cong \lambda /\left(2 \operatorname{Re} S_{n}\right)
$$

where $\lambda$ is the free-space wavelength.

\section{Antipodal Effect}

The as ymptotic approximation given above for $P_{\nu}(-\cos \theta)$ is not usable when $\theta$ is very near $\pi$. 
However, in this region, a modified form shows that the field is proportional to the Bessel function

$$
J_{0}\left[k a S_{n}(\pi-\theta)\right]
$$

which is finite at $\theta=\pi$ yet still behaves as

$$
(\sin \theta)^{-1 / 2} \cos \left[k a S_{n}(\pi-\theta)-(\pi / 4)\right]
$$

when the argument is large compared with unity.

\section{Near Field Behavior}

At very short distances (i.e., $\theta$ near 0 ), there is another expansion to the Legendre function which is quite useful. In the present notation, this result is given by

$$
\frac{P_{\nu}(-\cos \theta)}{\sin \nu \pi} \cong H_{0}^{(2)}\left(k S_{n} \rho\right)-\frac{1}{12}\left(\frac{\rho}{a}\right)^{2} H_{2}^{(2)}\left(k S_{n} \rho\right)+\text { terms in }(\rho / a)^{4},(\rho / a)^{6}, \text { etc. }
$$

where $\nu+\frac{1}{2}=k \rho S_{n}$ and $\rho=a \theta$. When the great circle distance $\rho$ is reasonably small compared with the earth's radius $a$, only the first term is important.

\section{General Field Representations}

For convenience in what follows, it is desirable to express the field components as a ratio to the quantity

$$
E_{o}=i(\eta / \lambda) I d s\left(e^{-i k \rho}\right) / \rho
$$

which is the radiation field of the source at a distance $\rho$ on a flat perfectly conducting earth. With this normalized factor, it is not difficult to show that

$$
E_{r}=E_{0} W
$$

where

$$
W=-i \pi(\rho / h) e^{i k \rho} \sum_{n=0}^{\infty} \Lambda_{n} S_{n}^{2} H_{0}^{(2)}\left(k S_{n} \rho\right)
$$

where terms containing $(\rho / a)^{2},(\rho / a)^{4}$, etc., have been omitted. An explicit formula for the excitation factor $\Lambda_{n}$ is given by [Wait, 1961],

$$
\Lambda_{n}=\frac{\delta_{n} C_{n}^{2}}{C_{n}^{2}-\Delta_{g}^{2}}
$$

where $\delta_{n}$ is obtained from

$$
\frac{1}{\delta_{n}}=1+i\left[\frac{\partial\left[R_{i}(C) R_{g}(C)\right] / \partial C}{2 k h R_{i}(C) R_{g}(C)}\right] C=C_{n} .
$$

In this equation,

$$
\begin{aligned}
& R_{g}(C)=\frac{C-\Delta_{g}}{C+\Delta_{g}}, \quad \Delta_{g} \cong \frac{1}{N_{g}}, \\
& R_{i}(C)=\frac{C-\Delta_{i}}{C+\Delta_{i}}, \quad \Delta_{i} \cong \frac{1}{N_{i}},
\end{aligned}
$$


and

$$
C_{n}=\left(1-S_{n}^{2}\right)^{1 / 2}
$$

The complex factors $C_{n}$ are solutions of the modal equation, which for ELF may be well represented by its flat-earth version. Explicitly, it is given by [Wait, 1962]

$$
R_{g}(C) R_{i}(C) \exp (-i 2 k h C)=\exp (-2 \pi i n)
$$

for $n=0,1,2, \ldots$ An approximated solution is given by (3) above or the simplified forms given by $(5 a)$ and $(5 b)$.

\section{Properties of the Modes}

The behavior of the modes in the earth-ionosphere waveguide is best understood by an examination of the complex function $W$ defined by (10). When $k p>>1$, corresponding to the "farzone," it follows from (10) that

$$
W \cong \frac{(\rho / \lambda)^{1 / 2}}{h / \lambda} \exp [i(2 \pi \rho / \lambda)-i \pi / 4] \sum_{n=0}^{\infty} \Lambda_{n} S_{n}^{3 / 2} \exp \left[-i 2 \pi S_{n} \rho / \lambda\right]
$$

where $\lambda$ is the free-space wavelength. Since $E_{r}=E_{0} W$, it follows that the vertical electric field of each of the modes is proportional to

$$
(1 / \rho)^{1 / 2} \exp \left[(2 \pi \rho / \lambda) \operatorname{Im} S_{n}\right] \exp \left[-i(2 \pi \rho / \lambda) \operatorname{Re} S_{n}\right]
$$

which have the character of outgoing cylindrical waves with damping according to $-(2 \pi / \lambda) \operatorname{Im} S_{n}$ nepers per unit distance. Actually, at ELF in the far zone, only the $n=0$ term is really important because of the evanescent character of the modes of order one and higher.

At short distances, where $2 \pi \rho / \lambda$ is comparable with unity, some of the evanescent modes are important. For this region, it appears that the only recourse is to sum the Hankel function series given by (10). Some results, using this approach, have been reported previously [Wait, 1962; Wait and Carter, 1960]. At very short distances, where $\rho<<h$, it becomes possible to neglect the presence of the ionosphere altogether. In this case,

$$
W \cong\left(1-\frac{i}{k \rho}-\frac{1}{k^{2} \rho^{2}}\right) \rightarrow-\frac{1}{k^{2} \rho^{2}}
$$

which shows that the vertical electric field varies according to $1 / \rho^{3}$ when $(2 \pi \rho / \lambda)$ is small compared with unity.

\section{Properties of the Excitation Factor}

Some comments regarding the excitation factor are in order. By carrying out the differentiations indicated in (11), it follows that

$$
\Lambda_{n}=\left[\frac{C_{n}^{2}}{C_{n}^{2}-\Delta_{g}^{2}}\right] \frac{1}{\left[1+\frac{i \Delta_{g}}{k h\left(C_{n}^{2}-\Delta_{g}^{2}\right)}+\frac{i \Delta_{i}}{k h\left(C_{n}^{2}-\Delta_{i}^{2}\right)}\right]},
$$

which is quite general provided that $\Delta_{i}$ and $\Delta_{g}$ are independent of $C$. In addition, it is assumed that $\left|\Delta_{g}\right|<<\left|\Delta_{i}\right|$ which is certainly valid at ELF. Equation (15) then simplifies to 


$$
\Lambda_{n}=\frac{1}{1+\frac{\sin 2 k h C_{n}}{2 k h C_{n}}},
$$

where use has been made of the mode equation (12). A further simplification, which is sometimes used at ELF, corresponds to using

$$
\Lambda_{0}=\frac{1}{2} \text { since } k h C_{0}<<1
$$

and

$$
\Lambda_{n}=1 \text { for } n=1,2,3, \ldots \quad \text { since } 2 k h C_{n} \cong 2 n \pi \text {. }
$$

The latter approximation is not very good unless $R_{i}$ has a magnitude near one. However, the error introduced by its use is negligible since these modes are evanescent in any case.

\section{Height-Gain Effects}

In the above discussion, the transmitting and the receiving antenna have both been assumed to be located on the earth's surface. The' effect of raising either or both of these is readily accounted for by the use of a height-gain function $F_{n}(z)$. For example, the vertical electric field of mode $n$ at height $z$, is $F_{n}(z)$ times the vertical electric field at height zero. By following through the formal derivation or by direct reasoning, it is found that

$$
F_{n}(z)=\frac{\exp \left(i k C_{n} z\right)+R_{g}\left(C_{n}\right) \exp \left(-i k C_{n} z\right)}{1+R_{g}\left(C_{n}\right)} .
$$

This obviously has the proper $z$-dependence, being the superposition of a downgoing and an upgoing wave. Furthermore, it has the required property that $F_{n}(0)=1$. In fact, a series development shows that

$$
F_{n}(z) \cong 1+i \Delta_{g} k z-\frac{\left(k C_{n} z\right)^{2}}{2}+\text { terms in }(k z)^{3},(k z)^{4}, \text { etc. }
$$

\section{Generalized Representation}

A representation for $E_{z}$ which is applicable at very short, intermediate, and great distancès may be obtained by generalizing (10), taking into account the $(\sin \theta)^{-1 / 2}$ dependence which is also consistent with (7). Also, the appropriate height-gain functions are inserted to account for the finite height $z_{0}$ of the transmitting antenna and the finite height $z$ of the receiving antenna (assuming that both are equivalent to vertical electric dipoles). Thus,

$$
E_{r}=E_{0} W\left(\frac{\theta}{\sin \theta}\right)^{1 / 2}
$$

where

$$
W=-i \pi(\rho / h) e^{i k \rho} \sum_{n=0}^{\infty} \Lambda_{n} S_{n}^{2} H_{0}^{(2)}\left(k S_{n} \rho\right) F_{n}\left(z_{0}\right) F_{n}(z)
$$

where $\rho=a \theta$. This result should be valid for all angular distances $\theta$ up until $\theta$ is near $\pi$. The heights $z$ and $z_{0}$ are to be restricted to the range 0 to $h$.

For the same idealized model as described above, the horizontal electric field $E_{\theta}$ of the vertical 
dipole source may be written

$$
E_{\theta}=-E_{0} S\left(\frac{\theta}{\sin \theta}\right)^{1 / 2}
$$

where

$$
S=\frac{\pi \rho}{h} e^{i k \rho} \sum_{n=0}^{\infty} \Lambda_{n} S_{n} H_{1}^{(2)}\left(k S_{n} \rho\right) F_{n}\left(z_{0}\right) G_{n}(z),
$$

with

$$
G_{n}(z)=C_{n}\left[\frac{\exp \left(i k C_{n} z\right)-R_{g}\left(C_{n}\right) \exp \left(-i k C_{n} z\right)}{1+R_{g}\left(C_{n}\right)}\right]
$$

It is also found that the magnetic field, which has only a $\phi$ component, is given by

$$
H_{\phi}=-E_{0} T\left(\frac{\theta}{\sin \theta}\right)^{1 / 2},
$$

where

$$
T=-\frac{\pi \rho}{h} e^{i k \rho} \sum_{n=0}^{\infty} \Lambda_{n} S_{n} H_{1}^{(2)}\left(k S_{n} \rho\right) F_{n}\left(z_{0}\right) F_{n}(z) .
$$

In summary, we see that the far-zone expressions for the field functions $W, S$, and $T$ may be written in the compact form

$$
\left[\begin{array}{l}
W \\
S \\
T
\end{array}\right]=\frac{(\rho / \lambda)^{1 / 2}}{(h / \lambda)} e^{i 2 \pi \rho / \lambda} e^{-i \pi / 4} \sum_{n=0}^{\infty} \Lambda_{n} e^{-i 2 \pi S_{n} \rho / \lambda}\left[\begin{array}{r}
S_{n}^{3 / 2} F_{n}(z) \\
-S_{n}^{1 / 2} G_{n}(z) \\
S_{n}^{1 / 2} F_{n}(z)
\end{array}\right] F_{n}\left(z_{0}\right),
$$

which is valid for $(\rho / \lambda)>>1$.

It is important to note that, quite generally, the ratio $S$ to $T$, for $z=0$, is $\Delta_{g}$. This is a necessary consequence of the boundary condition $E_{\theta}=-\eta \Delta_{g} H_{\phi}$ on the earth's surface. It is also interesting to note the ratio $W / T$ or $-E_{r} /\left(\eta H_{\phi}\right)$ is only approximately unity, being equal to $S_{n}$ in the far zone. At the shorter distances, when $\rho<\lambda$, the ratio $W / T$ will depart significantly from unity. Calculations which illustrate this phenomenon are available in the forms of curves of $W / T$ as a function of distance [Wait and Carter, 1960].

It is possible to excite ELF electromagnetic waves by other than a vertical electric dipole. For example, a horizontal electric dipole of moment $I d s$ and oriented in the $\phi=0$ direction will give rise to a vertical electric field $E_{r}$ according to the relation [Wait, 1962]

$$
E_{r}=E_{0} \hat{S}\left(\frac{\theta}{\sin \theta}\right)^{1 / 2} \cos \phi
$$

where

$$
\hat{S}=\frac{\pi \rho}{h} e^{i k \rho} \sum_{n=0}^{\infty} \Lambda_{n} S_{n} H_{1}^{(2)}\left(k S_{n} \rho\right) G_{n}\left(z_{0}\right) F_{n}(z)
$$

The function $\hat{S}$ has the same form as $S$ defined by (21) except that $z$ and $z_{0}$ are interchanged. The function $G_{n}\left(z_{0}\right)$ is defined by (22) which is valid for $z_{0}$ in the range 0 to $h$. Of some interest is 
the case where the horizontal electric dipole is buried to depth $z=-z_{1}$. Then, for the same dipole current moment, $G_{n}\left(z_{0}\right)$ is replaced by $\tilde{G}_{n}\left(z_{1}\right)$ where

$$
\tilde{G}_{n}\left(z_{1}\right) \cong \exp \left[-i\left(2 \pi z_{1} / \lambda\right)\left(N_{g}^{2}-S_{n}^{2}\right)^{1 / 2}\right] \cong \exp \left[-i\left(2 \pi z_{1} / \lambda\right) N_{g}\right]
$$

where $N_{g} \cong\left(\frac{1-i}{\sqrt{2}}\right)\left(\frac{\sigma_{g}}{\epsilon \omega}\right)^{1 / 2}$ is the refractive index of the earth which is assumed homogeneous.

The horizontal electric dipole also radiates horizontally polarized waves even when the ionosphere is isotropic. As indicated previously [Wait, 1961], this gives rise to a radiated $H_{r}$ component given by

$$
\eta H_{r}=-\sin \phi E_{0} T_{n}
$$

where

$$
T_{n}=-\frac{\pi \rho}{h} e^{i k \rho} \sum_{m=0}^{\infty} \Lambda_{m}^{h} S_{m} H_{1}^{(2)}\left(k_{\$} S_{m} \rho\right) F_{m}^{h}\left(z_{0}\right), F_{m}^{h}(z)
$$

where

$$
\Lambda_{m}^{h}=\frac{C_{m}^{2}}{C_{m}^{2}-\left(\Delta_{g}^{h}\right)^{2}} \frac{1}{1+\frac{i \Delta_{g}^{h}}{k h\left[C_{m}^{2}-\left(\Delta_{g}^{h}\right)^{2}\right]}+\frac{i \Delta_{i}^{h}}{k h\left[C_{m}^{2}-\left(\Delta_{i}^{h}\right)^{2}\right]}},
$$

in analogy to (15) for $\Lambda_{m}$. In the case of the horizontally polarized waves, the $C_{m}$ 's are solutions of the modal equation for TE (transverse electric) modes. It is given by

$$
R_{g}^{h}(C) R_{i}^{h}(C) \exp (-i 2 k h C)=\exp (-2 \pi i n)
$$

where

$$
R_{g}^{h}=\frac{C-\Delta_{g}^{h}}{C+\Delta_{g}^{h}} \text { and } R_{i}^{h}=\frac{C-\Delta_{i}^{h}}{C+\Delta_{i}^{h}}
$$

The appropriate height-gain function is given by

$$
F_{m}^{h}(z)=\frac{e^{i k C_{m} z}+R_{g}^{h}\left(C_{m}\right) e^{-i k C_{m}^{z}}}{1+R_{g}^{h}\left(C_{m}\right)}
$$

for $0<z<h$.

While the attenuation rate for the horizontally polarized modes, above cutoff, is quite low, the excitation factor $\Lambda_{m}^{h}$ is very small as indicated by the approximation

$$
\Lambda_{m}^{h} \cong-C_{m}^{2} /\left(\Delta_{g}^{h}\right)^{2} \cong-\Delta_{g}^{2} C_{m}^{2}
$$

On the other hand, the height-gain function is a rapidly increasing function of height. Thus,

$$
F_{m}^{h}(z) \cong 1+i k z / \Delta_{g}-\left(k C_{m} z\right)^{2} / 2+\ldots
$$

where, as usual, $k=2 \pi / \lambda$. Thus, for a highly elevated horizontal electric dipole, the received horizontally polarized field may be appreciable. 


\section{Cavity Resonances}

Within the framework of the present theory it is interesting to see how the Schumann resonance phenomenon emerges. The starting point is the expansion formula [Schumann, 1957].

$$
\frac{P_{\nu}(-x)}{\sin \nu \pi}=-\frac{1}{\pi} \sum_{n=0}^{\infty} P_{n}(x) \frac{2 n+1}{n(n+1)-\nu(\nu+1)},
$$

where the summation is over integral values of $n$. Introducing this result into (1) readily leads to the result

$$
E_{r}=\frac{i \operatorname{Ids} \nu(\nu+1)}{4 \pi a^{2} \epsilon \omega h} \sum_{n=0}^{\infty} P_{n}(x) \frac{2 n+1}{n(n+1)-\nu(\nu+1)}
$$

where $x=\cos \theta$. The early terms of the series are then proportional to

$$
\begin{aligned}
& P_{0}(x)=1, \\
& P_{1}(x)=\cos \theta, \\
& P_{2}(x)=\frac{1}{2}\left(3 \cos ^{2} \theta-1\right) .
\end{aligned}
$$

The $n=0$ term corresponds to quasi-static field configuration and it is of no particular interest in the present discussion. The terms corresponding to $n=1,2,3, \ldots$ are directly related to the Schumann cavity resonances. This may be demonstrated in a fairly simple manner. For a homogeneous isotropic ionosphere,

$$
\Delta_{i}=\frac{1}{N_{i}}=\left(\frac{i \epsilon \omega}{\sigma_{i}}\right)^{1 / 2}
$$

in terms of the conductivity $\sigma_{i}$ of the ionized medium. Then, using the relation of the operational calculus, $i \omega$ is replaced by $p$, which leads readily to the relation

$$
(c / a)^{2} \nu(\nu+1) \cong-p^{2}-p^{3 / 2} \alpha,
$$

where $\alpha=1 /\left[h\left(\sigma_{i} \mu\right)^{1 / 2}\right]$. Assuming that the current moment is an impulsive function of time,

$$
I d s=(I d s)_{0} \delta(t)
$$

where $\delta(t)$ is the unit impulse function at $t=0$. The Laplace transform of the source moment is then given by

$$
\int_{0}^{\infty} I d s e^{-p t} d t=(I d s)_{0}
$$

It is now a simple matter to show that the transform of the field is given by

$$
E_{r}(p)=\frac{(I d s)_{0}}{h^{2} C_{e}}\left(p+\alpha p^{1 / 2}\right) \sum_{n=0}^{\infty} P_{n}(x) \frac{2 n+1}{\omega_{n}^{2}+p^{2}+\alpha p^{3 / 2}},
$$

where

$$
\omega_{n}^{2}=(c / a)^{2} n(n+1),
$$


and

$$
C_{e}=\frac{4 \pi a^{2} \epsilon}{h}
$$

The actual time response $e_{r}(t)$ is now found from

$$
E_{r}(p)=\int_{0}^{\infty} e_{r}(t) e^{-p t} d t
$$

which may be inverted using standard techniques in operational calculus.

For small damping, it is found [Wait, 1962] that

$$
\begin{gathered}
e_{r}(t) \cong \frac{(I d s)_{0}}{h^{2} C_{e}} \sum_{n=0}^{\infty} P_{n}(x)(2 n+1) e^{-\Omega_{n} t} \cos \omega_{n}^{\prime} t, \\
\omega_{n}^{\prime} \cong \omega_{n}\left(1-\frac{\alpha}{2^{3 / 2} \omega_{n}^{1 / 2}}\right),
\end{gathered}
$$

and

$$
\Omega_{n}=\frac{\alpha \omega_{n}^{1 / 2}}{2^{3 / 2}}
$$

This transient result is valid for $\alpha^{2} \mathrm{t}>>1$ or $\mathrm{t}>>1 /\left(h^{2} \sigma_{i} \mu\right)$ and, therefore, it is only us able for sufficiently small damping. Actually, in the case of zero damping, $\alpha=0$, and $\omega_{n}^{\prime} \cong \omega_{n}$, which includes the static field for $\omega_{0}=0$, and the cavity modes $\omega_{1}, \omega_{2}, \omega_{3}, \ldots$ For $a=6400 \mathrm{~km}$,

$$
\begin{aligned}
& \omega_{1} / 2 \pi=10.6 \mathrm{c} / \mathrm{s}, \\
& \omega_{2} / 2 \pi=18.3 \mathrm{c} / \mathrm{s}, \\
& \omega_{3} / 2 \pi=25.9 \mathrm{c} / \mathrm{s} .
\end{aligned}
$$

The finite conductivity of the ionospheric shell tends to reduce these frequencies by about 20 percent.

\section{Final Remarks}

In the present theoretical exposition, a very simple model has been assumed, namely, a spherical homogeneous earth with a concentric homogeneous isotropic ionosphere. In recent years, there has been much effort devoted to more complicated models. Since this is a short review paper it is not possible to develop these generalizations and extensions. How ever, for the sake of completeness, the required modifications to the simple theory are briefly mentioned.

The presence of the earth's magnetic field renders the ionosphere anisotropic. Fortunately, when the earth's magnetic field is steeply dipping it is appropriate to invoke the QL (quasi-longitudinal) approximation. With this idealization, it is possible to say [Wait, 1962] that for an individual mode,

$$
\frac{\text { Attenuation rate with magnetic field }}{\text { Attenuation rate without magnetic field }} \cong \frac{\cos (\tau / 2)}{(\cos \tau)^{1 / 2}} \geqslant 1 \text {, }
$$

$$
\text { where } \tan \tau=\frac{\omega_{L}}{\nu}=\frac{\text { Longitudinal component of gyrofrequency }}{\text { Collision frequency }} \text {. }
$$

While this appears to be an exceptionally simple prescription, it does tally with current ideas on the subject as discussed by Galejs [1963] in his excellent article. It is seen that for $\tau>0$, the 
effect of the earth's magnetic field is to increase the attenuation and, consequently, the damping of the Schumann resonances are increased.

Finally, one should mention again that the nonsharpness of the lower edge of the ionosphere will play an important role in ELF propagation [Wait, 1962]. As discussed by Galejs [1963], this will modify considerably the frequency characteristic of predictions based on the simple homogeneous model. Another factor considered by both Fligel [1962] and Galejs [1963] is the influence of heavy ions which will modify the effective refractive index of the medium to some extent.

\section{Appendix. Application of Perturbation Theory to the Nonuniform Earth- Ionosphere Cavity}

In the main text of this paper, and in all published theoretical work on the Schumann resonance theory, the earth and the ionosphere are assumed to be concentrically stratified. In this appendix, it is proposed to consider the effect of nonuniformities of the ionosphere. In particular, by adapting a rather simple model it is shown how the resonant frequencies of the cavity could be modified by localized nonuniformities. The theory is somewhat analogous to that developed for microwave cavities under the influence of wall imperfections [Harrington, 1961].

We consider a concentric spherical cavity of inner radius $a$ and outer radius $a+h$. The surface impedance of the inner surface is zero, while that of the outer surface is $Z$, which is taken to be constant everywhere. The resonance frequencies for this situation are denoted $\omega_{n}$ where $n=1,2,3, \ldots$ and the associated electric and magnetic fields within the cavity are $\mathbf{E}$ and $\mathbf{H}$, respectively. We now consider that the surface impedance $Z$ of the outer surface is changed to $Z^{\prime}$ such that the difference $Z^{\prime}-Z$ may be a function of $\theta$ and $\phi$ in the spherical coordinate system. The resonance frequencies are now changed to $\omega_{n}^{\prime}$. The associated electric and magnetic fields are $\mathbf{E}^{\prime}$ and $\mathbf{H}^{\prime}$, respectively.

Maxwell equations for the unmodified cavity are:

and

$$
-\nabla \times \mathbf{E}=i \mu \omega_{n} \mathbf{H}
$$

$$
\nabla \times \mathbf{H}=i \epsilon \omega_{n} \mathbf{E},
$$

while, for the modified system,

and

$$
-\nabla \times \mathbf{E}^{\prime}=i \mu \omega_{n}^{\prime} \mathbf{H}^{\prime},
$$

$$
\nabla \times \mathbf{H}^{\prime}=i \epsilon \omega_{n}^{\prime} \mathbf{E}^{\prime}
$$

By making use of the vector identity,

$$
\nabla \cdot(\mathbf{A} \times \mathbf{B})=\mathbf{B} \cdot \nabla \times \mathbf{A}-\mathbf{A} \cdot \nabla \times \mathbf{B},
$$

it is now a simple matter to show that

$$
\nabla \cdot\left[\mathbf{H}^{\prime} \times \mathbf{E}-\mathbf{H} \times \mathbf{E}^{\prime}\right]=i\left(\omega_{n}^{\prime}-\omega_{n}\right)\left[\epsilon \mathbf{E} \cdot \mathbf{E}^{\prime}-\mu \mathbf{H} \cdot \mathbf{H}^{\prime}\right]
$$

By integrating (A6) over the volume $V$ of the cavity and making use of the divergence theorem, it follows that

$$
\iint_{A}\left[\mathbf{H}^{\prime} \times \mathbf{E}-\mathbf{H} \times \mathbf{E}^{\prime}\right] \cdot \mathbf{n} d a=i\left(\omega_{n}^{\prime}-\omega_{n}\right) \iiint_{V}\left[\boldsymbol{\epsilon} \mathbf{E} \cdot \mathbf{E}^{\prime}-\mu \mathbf{H} \cdot \mathbf{H}^{\prime}\right] d v,
$$


where $\mathbf{n}$ is the outward unit normal vector to the surface $A$ which bounds the volume $V$. Now, the boundary conditions on the outer surface are

$$
\mathbf{n} \times \mathbf{E}=Z \mathbf{H}_{t},
$$

and

$$
\mathbf{n} \times \mathbf{E}^{\prime}=Z^{\prime} \mathbf{H}_{t}^{\prime},
$$

where $\mathbf{H}_{t}$ and $\mathbf{H}_{t}^{\prime}$ are the tangential magnetic fields for the unmodified and the modified system. Because of the assumed perfect conductivity of the inner surface, that part of the surface integral in (A7) over $A$ vanishes. Therefore, by using (A8) and (A9), the general relation (A7) simplifies to

$$
\iint_{A}\left(Z^{\prime}-Z\right) \mathbf{H}_{t} \cdot \mathbf{H}_{t}^{\prime} d a=i\left(\omega_{n}^{\prime}-\omega\right) \iiint_{V}\left[\epsilon \mathbf{E} \cdot \mathbf{E}^{\prime}-\mu \mathbf{H} \cdot \mathbf{H}^{\prime}\right] d v,
$$

where the area $A$ is the outer surface of the concentric resonator.

Equation (A10) is an exact formulation of the problem. Thus, if the solution for the uniform cavity is known, this equation is a connecting relation between the fields for the modified cavity in terms of the change $Z^{\prime}-Z$ of the surface impedance of its walls. Unfortunately, it is not an explicit formula. Instead, it is a rather complicated integral equation. Nevertheless, if the modifications in the cavity are small it can be expected that the fields $\mathbf{E}^{\prime}, \mathbf{H}^{\prime}$ do not differ appreciably from $\mathbf{E}, \mathbf{H}$. Thus, the first-order perturbation formula for the change of resonant frequency of mode $n$ is given by

$$
\omega_{n}^{\prime}-\omega_{n}=\frac{i \int_{A} \int\left(Z^{\prime}-Z\right) \mathbf{H}_{t} \cdot \mathbf{H}_{t} d a}{\iint_{V} \int[\mu \mathbf{H} \cdot \mathbf{H}-\epsilon \mathbf{E} \cdot \mathbf{E}] d v}
$$

where $Z^{\prime}-Z$ is the specified change of surface impedance and the field quantities are all assumed known. Implicit in the statement of (A11) is the neglect of coupling between modes of different order. For example, if the uniform cavity is oscillating in a single mode, it is possible for additional modes to be excited when the wall impedance is modified in a localized region.

Here, we shall employ (A11) with some rather idealized assumptions about the nature of the fields in the uniform or unperturbed cavity. Specifically, we assumed that the unperturbed cavity is excited by a symmetrical source (e.g., radially oriented electric dipole) at $\theta=0$. From section 11 , it is seen that the fields associated with this mode are then given by

$$
E_{r} \cong A_{n}\left(\omega_{n}\right) P_{n}(\cos \theta)
$$

and

$$
\eta H_{\phi} \cong-i A_{n}\left(\omega_{n}\right) P_{n}^{1}(\cos \theta)\left(\frac{c}{\omega_{n} a}\right)
$$

where $A_{n}\left(\omega_{n}\right)$ is the amplitude of the mode at angular frequency $\omega_{n}$. The other field components are either zero or vanishingly small.

Using (A12) and (A13) in conjunction with (A10), it is found, without difficulty, that

$$
\begin{aligned}
-\left(\frac{c}{\omega_{n} a}\right) \frac{b^{2}}{\eta^{2}} \int_{0}^{2 \pi} \int_{0}^{\pi}\left[P_{n}(\cos \theta)\right]^{2} \sin \theta\left(Z^{\prime}-Z\right) d \theta d \phi & \\
& \cong i \epsilon\left(\omega_{n}^{\prime}-\omega_{n}\right) \int_{a}^{b} \int_{0}^{2 \pi} \int_{0}^{\pi}\left\{\left[P_{n}(\cos \theta)\right]^{2}+\left[P_{n}^{1}(\cos \theta)\right]^{2}\left(\frac{c}{\omega_{n} a}\right)^{2}\right\} r^{2} \sin \theta d \theta d \phi d r .
\end{aligned}
$$


Without introducing appreciable error, $r$ on the right-hand side of (A14) may be replaced by $b$. Then, by making use of the identities

$$
\int_{0}^{\pi}\left[P_{n}(\cos \theta)\right]^{2} \sin \theta d \theta=\frac{2}{2 n+1},
$$

and

$$
\int_{0}^{\pi}\left[P_{n}^{1}(\cos \theta)\right]^{2} \sin \theta d \theta=\frac{2 n(n+1)}{2 n+1}
$$

it follows, after some slight rearranging, that

$$
\omega_{n}^{\prime}-\omega_{n}=\frac{\left(\frac{i c}{2 \pi h}\right)\left[\frac{2 n+1}{2 n(n+1)}\right]}{\left[\left(\frac{\omega_{n} a}{c}\right)^{2} \frac{1}{n(n+1)}+1\right]} \int_{0}^{2 \pi} \int_{0}^{\pi} \delta(\theta, \phi)\left[P_{n}^{1}(\cos \theta)\right]^{2}(\sin \theta) d \theta d \phi
$$

where $\delta(\theta, \phi)=\left(Z^{\prime}-Z\right) / \eta$.

A very simple illustration of the perturbation formula is to let $Z=0$, which means that the unperturbed cavity has perfectly conducting walls. For this special case, we also take $Z^{\prime}$ independent of $\theta$ and $\phi$. In other words,

$$
\delta(\theta, \phi)=Z^{\prime} / \eta=\Delta
$$

where $\Delta$ is a constant. Then, by making use of (42b) and (A16), it follows that

$$
\omega_{n}^{\prime}-\omega_{n} \cong \frac{i \Delta c}{2 h}
$$

where

$$
\omega_{n}=(c / a)[n(n+1)]^{1 / 2} .
$$

This formula for $\omega_{n}^{\prime}$ is a remarkably simple result. Its validity may be tested by comparing it with the result based on a direct solution of the modal equation for the uniform concentric resonator. From such a previous analysis, it was shown that [Wait, 1964]

$$
\omega_{n}^{\prime}=\omega_{n}\left[1-i \frac{c \Delta}{\omega_{n}^{\prime} h}\right]^{-1 / 2}
$$

where $\omega_{n}$ is defined by (A19) and $\Delta$ is the (normalized) surface impedance at frequency $\omega_{n}^{\prime}$. It is easy to verify that (A20) reduces to (A18) when $c \Delta /\left(\omega_{n} h\right) \ll 1$. As expected, the first-order perturbation theory is only valid when the frequency $\omega_{n}^{\prime}$, for the modified system, is not too different from the frequency $\omega_{n}$ of the unmodified system.

Equation (A17) may be used to study the influence of a small localized disturbance. For example, we imagine that $Z^{\prime}$ is different from $Z$ only over a small region bounded in $\theta$ by $\theta_{0}-\left(\delta \theta_{0} / 2\right)$ and $\theta_{0}+\left(\delta \theta_{0} / 2\right)$, and bounded in $\phi$ by $\phi_{0}-\left(\delta \phi_{0} / 2\right)$ and $\phi_{0}+\left(\delta \phi_{0} / 2\right)$. The resonant frequency $\omega_{n}$ for the unperturbed cavity is to be found from [Wait, 1964]

$$
\omega_{n}=\omega_{0 n}\left[1-i \frac{c \Delta}{\omega_{n} h}\right]^{-1 / 2},
$$


where $\omega_{0 n}=(c / a)[n(n+1)]^{1 / 2}$, and where $\Delta=Z / \eta$ is a constant. Solving (A21) as a quadratic in $\omega_{n}$, it is seen that

$$
\omega_{n}=\omega_{0 n}\left[1-\frac{1}{4 \omega_{0 n}^{2}}\left(\frac{c \Delta}{h}\right)^{2}\right]^{1 / 2}+\frac{i c \Delta}{2 h}
$$

which approximates to

$$
\omega_{n} \cong \omega_{0 n}+\frac{i c \Delta}{2 h}
$$

if $\frac{c \Delta}{2 h \omega_{0 n}} \ll 1$. In terms of $\omega_{n}$ which is known, the resonant frequency $\omega_{n}^{\prime}$ for the perturbed resonant frequency $\omega_{n}^{\prime}$ is now given by

$$
\omega_{n}^{\prime}-\omega_{n} \cong \frac{\left(\frac{i c}{2 \pi h}\right)\left[\frac{2 n+1}{2 n(n+1)}\right]}{\left[1+\frac{\omega_{n}^{2}}{\omega_{0 n}^{2}}\right]} \bar{\delta}\left(\delta \theta_{0}\right)\left(\delta \phi_{0}\right)\left[P_{n}^{1}\left(\cos \theta_{0}\right)\right]^{2} \sin \theta_{0},
$$

where $\bar{\delta}$ is the average value of $\delta(\theta, \phi)$ over the small perturbed region. The explicit $\theta_{0}$ dependence of the right-hand side of (A24), for the first two modes, is evident from the identities

$$
\begin{aligned}
& {\left[P_{1}^{1}\left(\cos \theta_{0}\right)\right]^{2}=\sin ^{2} \theta_{0},} \\
& {\left[P_{2}^{1}\left(\cos \theta_{0}\right)\right]^{2}=9 \sin ^{2} \theta_{0} \cos ^{2} \theta_{0} .}
\end{aligned}
$$

These show that the first mode is most affected by surface impedance perturbations near the equatorial plane whereas the second mode is most affected by perturbations near $\theta_{0}= \pm 45^{\circ}$.

It would appear that the perturbation theory of cavity resonators should be a useful approach to investigating the influence of localized nonuniformities of the ionosphere on the Schumann-type resonances.

\section{References}

Brock-Nannestad, L. (1 Sept. 1962), Bibliography of electromagnetic phenomena with special reference to ELF (1-3000 c/s), Tech. Report No. 10, SACLANT ASW Research Center, Viale San Bartolomeo 92, La Spezia, Italy.

Fligel, D. S. (1962), Properties of the index of refraction, attenuation coefficient, and transmission coefficient of the ionosphere at low and very low frequencies (1 to $10^{6} \mathrm{c} / \mathrm{s}$ ), Geomagnetism and Aeronomy 2, 886-903 (Moscow).

Galejs, J. (July 1963), Terrestrial extremely low frequency propagation, paper presented at the NATO Advanced Study Institute on Low Frequency Electromagnetic Radiation, Bad Homburg, Germany. See also paper by J. Galejs in this issue.

Harrington, R. F. (1961), Time harmonic electromagnetic fields (McGraw-Hill Book Co., New York, N.Y.).

Schumann, W. O. (1957), Uber elecktrische Eigenschwingungen des Hohlraumes Erde-Luft-Ionosphare arregt durch Blitzentladungen, Zeits. angew. Phys. 9, 973.

Wait, J. R. (Sept.-Oct. 1961), A note concerning the excitation of ELF electromagnetic waves, J. Res. NBS 65D (Radio Prop.), No. 5, 481-484.

Wait, J. R. (1962), Electromagnetic waves in stratified media (Pergamon Press, Oxford, and Macmillan Co., New York, N.Y.). Wait, J. R. (Apr. 1964), On the theory of Schumann resonances in the earth-ionosphere cavity, Can. J. Phys. 42, 575-582. Wait, J. R., and N. F. Carter (Mar. 1960), Field strength calculations for ELF radio waves, NBS Technical Note No. 52 (PB-161553).

(Paper 69D8-539) 